\title{
Contents to Volume 124 (2000)
}

\section{Volume 124 No. 1, October 2000}

Marek Golasiński: On Injective and Cofibrant Equivariant Minimal Models

Stephen Debacker: Homogeneity of Certain Invariant Distributions on the Lie Algebra of $p$-adic $\mathrm{GL}_{n}$

B. Enriquez and G. Felder: Commuting Differential and Difference Operators Associated to Complex Curves I

Roland Dreier: Analytic Variation of $p$-adic Abelian Integrals

Antoine Chambert-Loir and Yuri Tschinkel: Points of Bounded Height on Equivariant Compactifications of Vector Groups, I

P. B. A. Lecomte and V. Yu. Ovsienko: Cohomology of the Vector Fields Lie Algebra and Modules of Differential Operators on a Smooth Manifold

Henrik Vosegaard: A Characterization of Quasi-Homogeneous Purely Elliptic Complete Intersection Singularities

\section{Volume 124 No. 2, November 2000}

Jonathan Lubin: Formal Flows on the Non-Archimedean Open Unit Disk

Rob de Jeu: Towards Regulator Formulae for the $K$-Theory of Curves over Number Fields

Bernhard Köck: Symmetric Powers of Galois Modules on Dedekind Schemes 
Volume 124 No. 3, December 2000

E. Muñoz Garcia: Fibrés d'intersection

Joachim Mahnkopf: Eisenstein Cohomology and the Construction of $p$-Adic Analytic $L$-Functions

Atsushi Shiho: A Note on Class Field Theory for Two-Dimensional Local Rings

$305-340$

Yoshinobu Kamishima: Holomorphic Torus Actions on Compact Locally Conformal Kähler Manifolds

Author Index to Volume 124 (2000)

Contents to Volume 124

$353-354$ 